\title{
Semantic model and optimization of creative processes at mathematical knowledge formation
}

\author{
Victor Egorovitch Firstov \\ Department of Mechanics and Mathematics, Saratov State University, Saratov, Russia; firstov1951@gmail.com
}

Received 26 February 2010; revised 24 April 2010; accepted 29 April 2010.

\begin{abstract}
The aim of this work is mathematical education through the knowledge system and mathematical modeling. A net model of formation of mathematical knowledge as a deductive theory is suggested here. Within this model the formation of deductive theory is represented as the development of a certain informational space, the elements of which are structured in the form of the orientated semantic net. This net is properly metrized and characterized by a certain system of coverings. It allows injecting net optimization parameters, regulating qualitative aspects of knowledge system under consideration. To regulate the creative processes of the formation and realization of mathematical knowedge, stochastic model of formation deductive theory is suggested here in the form of branching Markovian process, which is realized in the corresponding informational space as a semantic net. According to this stochastic model we can get correct foundation of criterion of optimization creative processes that leads to "great main points" strategy (GMP-strategy) in the process of realization of the effective control in the research work in the sphere of mathematics and its applications.
\end{abstract}

Keywords: The Cybernetic Conception; Optimization of Control; Quantitative and Qualitative Information Measures; Modelling; Intellectual Systems; Neural Network; Mathematical Education; the Control of Pedagogical Processes; Creative Pedagogics; Cognitive and Creative Processes; Informal Axiomatic Theory; Semantic Net; Net Optimization Parameters; The Topology of Semantic Net; Metrization; the System of Coverings; Stochastic Model of Creative Processes at the Formation of Mathematical Knowledge; Branching Markovian Process; Great Main Points Strategy (GMP-Strategy) of the Creative Processes Control; Interdisciplinary Learning: Colorimetric Barycenter

\section{INTRODUCTION}

"The book of nature is written by the mathematical language" [1]. This Galilei's manifesto determined the methodology of natural science development on the basis of observations and experiments, the result of these observations and experiments are interpreted within the frames of corresponding mathematical model. The latter implicitly assumes the mechanism of science integration thus, this methodology of development have been successfully realized during the last century beginning with famous Newton's "Mathematical Priciples of Natural Philosophy" (1687) [2]. The reason of this success is easily explained by L. Boltzmann's assertion in his book [3]:" The theory is more practical, it is quantum-essence of experiment." One can understand in the sence that quantum-essence is the system of postulates, which is in the foundation of theoretical model of the object under consideration.

In $1948 \mathrm{~N}$. Wiener [4] had stated main cybernetics' positions. He had done it on C. Shannon's information theory (1948) [5]. According to this theory, any process of control system represents some transformation of systemic information. Information is basis notion of cybernetics and has an abstract quantitative measure. It allows to measure both material and non-material aspects of the object under consideration. Thus, possibilities of mathematical modelling have extended including processes in social and humanitarian fields [6].

With the appearance of computers in the middle of the $\mathrm{XX}^{\text {th }}$ century, a separate direction connected with the realization of intellectual systems (IS) has been formed in cybernetics $[7,8]$. Accents in this case focused on learning of cognitive processes. In 1980s this led to the building of learning ACT-theory [9] and neural network associative model of J. Hopfild [10]. Modern level of IS development represents adaptive learning systems [11].

A half century experience of IS development shows that their possibilities are determined by mathematical model parameters, which are in the ground of IS. Thus, the questions of IS efficiency lead to the parameters op- 
timization of corresponding mathematical models which describe given cognitive processes. Formally, it assumes the investigation of topology of semantic nets which realize given cognitive processes. This investigation allows determining the system of net parameters with the help of which one can influence the knowledge system. Thus, optimal control by cognitive processes is carried out, including creative search and interdisciplinary learning.

\section{SEMANTIC MODEL OF INFORMAL AXIOMATIC THEORY}

Let $S=(M ; \Sigma)$ is a mathematical structure, where $M=$ $\left\{M_{1} ; \ldots ; M_{k}\right\}$ is a system of ground sets, representing main objects of structure $S$, and $\Sigma=\left\{\alpha_{1} ; \ldots ; \alpha_{s}\right\}$ is a system of axiom, describing ground relations between them. The informal theory $T h(S)$ of the structure $S$ represented denumerable set, the elements of which are ordered by definite rules of conclusion. One can speak of the theory $\operatorname{Th}(S)$ as of the structural space information [12], which is formed within given concluded rules in the form of constructively infinite recursive procedure, that is to say, the space $\operatorname{Th}(S)$ is some kind of Herbrand's universe analogue.

Theory space $T h(S)$ is given by the digraph $\vec{\Gamma}(S)$, representing the mathematical structure $\mathrm{S}$ in the form of semantic net, which realizes the passing of definite object information. At this, the set $\operatorname{Th}(S)$ determines the nodes of the object net domain $\vec{\Gamma}(S)$, and its arcs (orientated edges) are given with the help of the commutator I-set of functions $\mathrm{f}$ of following type:

$$
f: T_{1} ; \ldots ; T_{n} \rightarrow T
$$

where $T ; T_{1} ; \ldots: T_{n} \in T h(S)$, and symbol $\rightarrow$ means informal logical consequence of the statement $T$ from $T_{1} ; \ldots ; T_{n}$. Digraph $\vec{\Gamma}(S)$ is appeared by the pair $(V ; E)$, where the set of nodes $\mathrm{V}$ and the set of arcs $\mathrm{E}$ is defined by the expressions:

$$
V=T h(S) \cup I ; \quad E \subset(T h(S) \times I) \cup(I \times \bar{\Sigma}),
$$

where $\bar{\Sigma}$ is the complement of the system axioms $\Sigma$ till $T h(S)$, and, without community restrict, the axiom system $\Sigma$ may be considered independent. For a given digraph $\vec{\Gamma}(S)$, the system of nodes $\Sigma \subset T h(S)$ represents the sources and, thus, $\vec{\Gamma}(S)$ represents of the semantic net, which determines the information space structure of axiomatic theory $T h(S)$.

\section{TOPOLOGICAL PROPERTIES OF THE SEMANTIC NET $\vec{\Gamma}(S)$}

In order to investigate semantic net properties $\vec{\Gamma}(S)$, topological presentations, realized according to two directions, are used [12].

The first direction determines routes, distances and connection between subject nodes of the semantic net $\vec{\Gamma}(S)$. Let on the digraph $\vec{\Gamma}(S)$ (2) the nodes are distinguished $v_{0} ; v_{1} ; \ldots ; v_{n} \in V$, which form the sequence of $\operatorname{arcs}$

$$
\vec{L}\left(v_{0} ; \ldots ; v_{n}\right):\left(v_{0} ; v_{1}\right)\left(v_{1} ; v_{2}\right) \ldots\left(v_{n-1} ; v_{n}\right) \in E,
$$

where $\left(v_{i} ; v_{i+1}\right)=\left(v_{i} ; f_{i}\right)\left(f_{i} ; v_{i+1}\right) ; \quad f_{i} \in I ; i=\overline{0 ; n-1}$. Thus, the oriented route is given, connecting the node $v_{0}$ with $v_{n}$ (it means, that the node $v_{n}$ is reached from the node $v_{0}$ ), and the nodes $\mathrm{v}_{\mathrm{i}}, i=\overline{0 ; n-1}$, are called intermediate ones on the route (3), and this fact is expressed in the form of: $v_{0} \prec v_{i} \prec v_{n}$. The route length (3) and the distance from the node $v_{0}$ to the node $v_{n}$ are, correspondingly, determined by correlations:

$$
\left|\vec{L}\left(v_{0} ; \ldots ; v_{n}\right)\right|=n ;\left|\vec{r}\left(v_{0} ; v_{n}\right)\right|=\inf \left|\vec{L}\left(v_{0} ; v_{n}\right)\right|,
$$

where $\left|\vec{L}\left(v_{0} ; v_{n}\right)\right|$ is the set of all route lengths, connecting the node $v_{0}$ with $v_{n}$.

The second direction of semantic net $\vec{\Gamma}(S)$ investigations is carried out with the help of special system of coverings, which is formed by the following way. For arbitrary node $T \in T h(S)$ the set is determined:

$$
U(T)=\left\{T_{i} \mid T_{i} \prec T \underset{T h(S),}{\vee} T_{i}=T, T_{i} ; T \in T h(S), i \in N\right\} \underset{(5)}{\subset}
$$

the elements of which represent the nodes, for which the node $\mathrm{T}$ is reached on the digraph $\vec{\Gamma}(S)$. The set $U(T)$ is called the sphere of dominating of the node $\mathrm{T}$ in the space $T h(S)$, and its power $|U(T)|$ determines the capacity of the dominating sphere $\mathrm{U}(\mathrm{T})$. Capacity $|U(T)|$ in given case represents topological of information quantity according to N. Rashevsky [13]. The following topological properties are set up for dominating spheres:

1) $T_{1} \in U(T) \Leftrightarrow U\left(T_{1}\right) \subseteq U(T)$,

moreover, the equality $U\left(T_{1}\right)=U(T)$ at $T \neq T_{1}$ is equivalent to the fact, that the nodes $T ; T_{1}$ are linked by the cycle.

2) If $U\left(T_{1}\right) \cap U\left(T_{2}\right) \neq \varnothing$ and sets $U\left(T_{1}\right), U\left(T_{2}\right)$ are not linked by implication, then among nodes $T \in$ $U\left(T_{1}\right) \cap U\left(T_{2}\right)$, at least one of them, is a point of net branching $\vec{\Gamma}(S)$.

Let $\vec{L}(\Sigma ; T)$ is the set of routes, leading from axioms $\Sigma$ to the node $T$. Then with the help of (4), the distance from $\Sigma$ to $T$ and the diameter of the sphere $U(T)$, are correspondently, determined:

$$
|\vec{r}(\Sigma ; T)|=\inf |\vec{L}(\Sigma ; T)|, d(U(T))=\sup |\vec{L}(\Sigma ; T)| .
$$

Within the conception capacity (5) and distance (7), a recursive procedure is determined, for forming the sys- 
tem of inclusion coverings in the theory structure $T h(S)$.

Theorem 1. In the space $T h(S)$ one can always underline a denumerable assemblage of subsets, which form the chain

$$
T h(S) \supset T h_{1}(S) \supset T h_{2}(S) \supset \ldots,
$$

thus, the chain of inclusion coverings of the space $T h(S)$ is formed:

$$
h(S) \subset \square h_{1}(S) \subset \square h_{2}(S) \subset \ldots,
$$

where $\square h(S)=\{U(T): T \in T h(S)\}$,

$=\left\{U_{i}(T): T \in T h_{i}(S)\right\}$,

$T h_{i}(S)=\{T: T \in T h(S),|\vec{r}(\Sigma ; T)| \geq i\}, i=1 ; 2 ; \ldots$

Thus, algorithm of knowledge generalization is given in the semantic net $\vec{\Gamma}(S)$ : if a certain section $h_{i}(S)$ is chosen in chain (9), then an object sphere of knowledge $T h(S)$ is covered by the system of spheres $U_{i}(T) \in$ $h_{i}(S)$ and in each of such sphere the dominated node $T \in U_{i}(T)$ presents the generalization of the rest elements of sphere $U_{i}(T)$. As a result, the spheres $U_{i}(T)$ are classified by some system of characteristics in order to form an appropriate conception. Accordingly, the property (9) in the process of generalization realizes the principle of "matryoshki", and as a result the level of abstraction's conception is gradually increasing. It means that the process of getting knowledge is connected with the development of intellect.

\section{OPTIMIZATION OF THE DEDUCTIVE CONCLUSION ON SEMANTIC NETS}

Let among the statements $T \in T h(S)$ there is finite set of positions $T_{\mathrm{i}_{1}} ; \ldots ; T_{i_{k}}$, for which in the net $\vec{\Gamma}(S)$ there is the only function $f_{m} \in I$ with the range of definition Dom $f_{m}=\left\{T_{\mathrm{i}_{1}} ; \ldots ; T_{i_{k}}\right\}$. This function realizes the informal logical conclusion:

$$
f_{m}: T_{\mathrm{i}_{1}} ; \ldots ; T_{i_{k}} \rightarrow T \text {. }
$$

If Dom $f_{m} \not \subset \Sigma$, then each of the nodes $T_{\mathrm{i}_{1}} ; \ldots ; T_{i_{k}}$ $\in$ Dom $f_{m} \backslash \Sigma$ similarly (10) has a corresponding $I$-node and also occurs the result, one-valued following from corresponding positions of predicate universe $\operatorname{Th}(S)$ and so on, till we come to the proofs:

$$
f_{11}: \Sigma_{1} \rightarrow T_{1} ; f_{12}: \Sigma_{2} \rightarrow T_{2} ; \ldots ; f_{1 r}: \Sigma_{r} \rightarrow T_{\mathrm{r}},
$$

where $\Sigma_{1} ; \ldots ; \Sigma_{r} \subseteq \Sigma 5$. Thus, in general case, procedure's proof of the statement $T \in T h(S)$ is a partially ordered set $B(T)$, which is made up of predicate nodes, structured through functions (10),(11). It is evident, that $B(T) \subseteq$ $U(T)$ and, hence, the sphere of dominating $U(T)$ present a union of the all possible proofs' of the statement $T$.

Let $\vec{L}(\Sigma ; T)$ is a set of routes from the axioms $\Sigma$ to the node $\mathrm{T}$ in the proof $B(T)(10),(11)$. Then, the length $|\vec{b}(T)|$ of proof $B(T)$ presents critical way on $B(T)$ and is determined through recursion:

$$
|\vec{b}(T)|=\max \left(\left|\vec{b}\left(T_{\mathrm{i}_{1}}\right)\right| ; \ldots ;\left|\stackrel{b}{b}\left(T_{i_{k}}\right)\right|\right)+1=d(B(T)),
$$

where $d(B(T))$ - the diameter of the proof $B(T)$, which is determined similarly (7).

Apart from the length (12), the proof $B(T)$ is characterized by the capacity $|B(T)|$. Through regulation by these parameters of the conclusion the tasks of the optimization while forming the knowledge, in the form of the theory $\operatorname{Th}(S)$ are considered in the net $\vec{\Gamma}(S)$. Let $B_{1}(T) ; \ldots ; B_{j}(T)$ are different proofs of the statement $T \in$ $T h(S)$, having the lengths $\left|\vec{b}_{1}(T)\right| ; \ldots ;\left|\vec{b}_{j}(T)\right|$ and capacities $\left|B_{1}(T)\right| ; \ldots ;\left|B_{j}(T)\right|$. Then, the following tasks of the optimization are determined on the net $\vec{\Gamma}(S)$ :

$$
\begin{aligned}
& B_{0}(T)=\operatorname{opt}\left(B_{1}(T) ; \ldots ; B_{j}(T)\right) \Leftrightarrow\left|\vec{b}_{0}(T)\right|= \\
& \min \left(\left|\vec{b}_{1}(T)\right| ; \ldots ;\left|\vec{b}_{j}(T)\right|\right), \\
& B_{0}(T)=\operatorname{opt}\left(B_{1}(T) ; \ldots ; B_{1}(T)\right) \Leftrightarrow\left|B_{0}(T)\right|= \\
& \min \left(\left|B_{1}(T)\right| ; \ldots ;\left|B_{j}(T)\right|\right)
\end{aligned}
$$

Each of the tasks (13), (14) present the optimization of the statement proof $T \in T h(S)$, accordingly, by the minimization of its length or capacity. On the whole, these tasks may be considered together. This statement of optimal tasks means the simplification of the proof through reducing the volume of the analysed information. On the whole, it is coordinated with the principles of the information theory.

For example, the optimization of Pythagorean theorem proofs was carried out according to criteria (13); (14), through the analysis of existing variants in school geometry: Euclidean classical proof, the proofs of indian mathematician Bhascara and vectorial method of proof with the help of scalar product [12]. The parameters of the proofs of Pythagorean theorem $T$ in the Euclid's, Hillbert's and Weyl's axiomatics are given in the Table 1.

Table 1. Metric characteristics of main versions of proofs Pythagorean theorem in different axiom system.

\begin{tabular}{ccccc}
\hline Proof & Axiomatics & i & $\left|\vec{b}_{i}(T)\right|$ & 10 \\
\hline Euclid & Euclid (IV B.C.) & 0 & $1 B_{i}(T) \mid$ \\
\hline Bhascara (proof-I, $\sim 1150)$ & D. Hilbert (1899) & 1 & 9 & 36 \\
Bhascara (proof-II, $\sim 1150)$ & H. Weyl (1918) & 2 & 12 & 23 \\
vectorial method & 3 & 2 & 35 \\
\hline
\end{tabular}


As it is seen from the Table 1, the optimization of proofs according to the criteria (13), (14) the preference is on the side of the vectorial proof in Weyl's axiomatics. However, the rise of abstraction level happens in the teaching process, which is in the inverse dependence with didactic principles of accessibility and visual teaching aids. This fact has its reflection in Russian teaching literature on elementary geometry. The analysis of this literature for the period of 1768-2000 shows, that in the second half of the XIX ${ }^{\text {th }}$ century, Euclid's classical proof is practically nowhere met in school textbooks and Bhascara's proofs are mostly used, because they are more vivid and accessable [12].

\section{FORMALIZATION OF CREATIVE PROCESSES DURING THE ASSIMILATION OF THE SPACE $T h(S)$ AND THE RANGE SIGNIFICANCE OF THE ELEMENTS IN THE NET $\vec{\Gamma}(S)$}

One can regard creative processes in the training as the generalization of knowledge at the metalevel with the help of heuristics. The link between metalevel knowledge and the known object sphere of knowledge is formally expressed in the fact, that the space $T h(S)$ is formed with the help of endless recursive procedure and, hence, one can say of current statement $T h_{1}(S)$, for which $\Sigma \subset T h_{1}(S) \subset T h(S)$, then knowledge metalevel is introduced with the help of partition

$$
T h(S)=T h_{1}(S) \cup C T h_{1}(S),
$$

where $C T h_{1}(S)$ is the complement $\operatorname{Th}_{1}(S)$ till $\operatorname{Th}(S)$, which determines the knowledge metalevel in the form of nodes. To these nodes relation of incidence in the net $\vec{\Gamma}(S)$ is heurictically established. The partition (15) in the net $\vec{\Gamma}(S)$ induces set partition of functional nodes $I=I_{1} \cup C I_{1}$, where $C I_{1}$ is the set of functions, realizing the conclusion to metalevel:

$$
C I_{1}: T h_{1}(S) \rightarrow C T h_{1}(S) \text {. }
$$

Correlations (15) and (16) present a formalisational description of the knowledge generalization procedure to metalevel in the process of axsiomatical theory $\operatorname{Th}(S)$ formation. The optimization procedure of this process is forming in the range of presentation about node significance in the semantic net.

Let $U(T)$ is the sphere of dominating of the statement $T$ and $B_{1}(T) ; \ldots ; B_{n}(T)$ are possible proofs of this statement, having lengths $\left|\vec{b}_{1}(T)\right| ; \ldots ;\left|\vec{b}_{n}(T)\right|$. Let us call the quantity

$$
D(\Sigma ; T)=\min \left(\left|\vec{b}_{1}(T)\right| ; \ldots ;\left|\vec{b}_{n}(T)\right|\right)
$$

some logical distance from axiom's $\Sigma \subset T h(S)$ to the statement $T$. In fact, logical distance $D(\Sigma ; T)$ coincides with algorithmical determination of information quantity according to A.N. Kolmogorov [14].

Formally, any significance presents a partial order in the space $T h(S)$ and it is given in the form of domination relation according to Pareto:

$$
T_{1} \triangleleft T \Leftrightarrow|U(T)| \geq\left|U\left(T_{1}\right)\right| \wedge D\left(\sum ; T\right) \leq D\left(\Sigma ; T_{1}\right),
$$

where one of the inequalities is strictly done. In the case of defining (18), the statement $\mathrm{T}$ is considered to be significant than $T_{1}$ and it means that more important elements of the space $\operatorname{Th}(S)$ are more influential (the first inequality in (18)), and they are closer to the information system sources $\Sigma$ (the second inequality in (18)). One can also interpret the significant elements as huge main points of the net $\vec{\Gamma}(S)$, which are closer to its sources.

\section{GMP-OPTIMIZATION STRATEGY OF CREATIVE PROCESSES IN THE FORMATION OF MATHEMATICAL KNOWLEDGE}

The reasons of relationships significance (18) is carried out in the language of theory's random processes [15]. As in the process of creative searching the moments of revealing time t of new theory form statements $\operatorname{Th}(S)$ are not determined, then its creation presents a random process $T h(S ; t)$ with continuous time $t \geq 0$ and with denumerable set of the states, the moments of transititions between them are distributed in the interval $t>0$ by chance.

Theorem 2. The random process $T h(S ; t)$ is a heterogeneous branching Markovian process.

Heterogeneous in time, the branching Markovian process $\mathrm{Th}(\mathrm{S} ; \mathrm{t})$ is determined as a process, the transition probability $P_{i n}(\tau ; t)$ of which satisfy the KolmogorovChapman equation with branching condition:

$$
P_{i k}(\tau ; t)=\sum_{\mathrm{r}_{1}+\ldots+r_{i}=k+i(l-1)} P_{l r_{1}}(\tau ; t) P_{l r_{2}}(\tau ; t) \ldots P_{l r_{i}}(\tau ; t),
$$

where $P_{i k}(\tau ; t)$ is the probability of the condition having $i$ elements at the moment $\tau$, to the moment $t$ it will contain $k \geq i \geq l=|\Sigma|$ elements and the evolution $P_{i k}(\tau ; t)$ is described by Kolmogorov system of differential equations for the heterogeneous branching Markovian process [16].

The reason of Pareto-optimization procedure by significance criterion (18) means that when the information space theory $\operatorname{Th}(S)$ is mastered by chance and is realized by branching Markovian process $T h(S ; t)$ then more significant theory statements $T h(S)$ have higher probabilities of transitions between the process statements $\operatorname{Th}(S ; t)$ and the result of this is as follows: let $P_{i k}=P_{i k}(\tau ; t)$ is 
the solution of Kolmogorov equations under condition (19). That will be enough to examine an example $k=i+1$ and then from the branching condition (19) you will get:

$$
P_{i, i+1}=i P_{l, l+1} P_{l l}^{i-1}=i\left(1-P_{l l}\right) P_{l l}^{i-1} .
$$

With the increasing the length of the interval $(\tau ; t)$, the meaning $\mathrm{P}_{l l} \rightarrow 0$ and the function $P_{i, i+1}$ is increasing in the wide range $l \leq i<-\ln P_{l l}$. Since the meaning $\mathrm{i}$ in this case is linked with the quantity $T h(S)$, which corresponds to $i$-state of the process $T h(S ; t)$, then it goes without saying, that, under other similar conditions, the dominating sphere $U(T)$ with more capacity $|U(T)|$ has more chances to widen, because the probability of proof of new statements at forming theory $T h(S)$ is increasing. Thus, the reason of the first inequality in the Pareto-optimization procedure (18) is given. However, the growth of transition's probability $P_{i k}(\tau ; t)$ in this model occurs not only with the growth $\mathrm{i}$, but also with the decrease $\tau$, because in this case the interval length $(\tau ; t)$ is increasing. The $\tau$ decrease is equivalent to the decrease of logical distance (17) that leads to the reasons of second inequality in Pareto-optimization (18). Thus, the conception of the range significance of the statements theory $T h(S)$ by means of Pareto-domination (18) has a sufficient reason. According to this reason, the optimum control by creative processes in training comes to effective control of definite random process according to the criterion of significance (18). In this case, the effective strategy of theory formation $T h(S)$ in the creative process leads to the conception of "great main points" in the net $\vec{\Gamma}(S)$ or GMP-strategy. This conception intends the investigation, coming out from significant statements $T_{01} ; \ldots ; T_{0 k} \in T h(S)$, have been chosen according to the net criterion of the significance (18). The inductive hypothesis $\mathrm{H}$, given on the base of these statements, has more chances "to be materialized" as a logical generalization of starting positions. The idea of existing "great main points" is in tune with the modern psychological conceptions in the field of intellectual theory [17]. Ac- cording to these conceptions, "great main points", which have an increased sensitivity to certain semantic influence, can qualitatively change the character of understanding' problem situation.

\section{GMP-STRATEGY AND HILBERT'S MATHEMATICAL PROBLEMS}

A vivid illustration of optimization creative search within the framework of GMP-strategy presents the solution of well-known problems in the theory of numbers, and also Hilbert's problems (1900) [18]. The chronology of their position and solution is exactly known (Table 2).

The analysis shows, if the period of decision Goldbach's, Waring's and Fermat's problems in the theory of numbers makes up hundreds of years, then Hilbert's problems has a unique result, which occurs by 1-2 less. It is important to say, that the choice of 23 problems from a wide manifold of mathematical ones, appearing in the XIX-XX ${ }^{\text {th }}$ centuries according to Hilbert's report at the II International Congress of Mathematicians (1900), supposed quite certain "rules of selection". The sense of them is the realization GMP-strategy. Those problems are interesting, the decision of which is possible at a given level of mathematical development. These problems can give a further progress to mathematics.

\section{THE EXPERIMENT OF GMP-STRATEGY IN INTERDISCIPLINARY LEARNING}

The possibilities of GMP-strategy are not limited only by the optimization of the mathematical researches but are spread over the interdisciplinary training level within the framework of morphism's category. In this case, the author's experience shows, that the realization of GMP-strategy usually takes place on the basis of some

Table 2. The problematic optimization of the research work in mathematics.

\begin{tabular}{cccc}
\hline \multicolumn{1}{c}{ Problems } & Problem Decision & & Period of problem decision, \\
years
\end{tabular}


problems

general scientific methodology for example, in the course of a canon or the conception of centrism.

The conception of canon development is often observed in social sciences for example, in the states theory. As a canon here, the ideals of a democracy state justice can be taken into consideration dating back to the republic of Ancient Rome (510/509 B.C.) and Platon's dialogues (427-347 B.C.). In this case, GMP-strategy is started through the axiomatization of the justice canon, on the basis of which the deductive theory is formed.

Nowadays, this theory represents a separate sector in mathematics, known as cooperative games, within the framework of which, it became possible to explain the peculiarities of modern democracy [19].

The centrism conception represents a general principle of methodology, the grounds of which dates back to ancient times, having a reflection in antiquity doctrines, traditions and religions. According to Archimedean interpretation, this principle leads to the conception about center of gravity (barycenter) of a material corpse. On the ground of this principle, modern classical mechanics was formed. GMP-strategy on the basis of barycenter conception is formed by the following way. In 1827 , A.Möbius gave mathematical grounds to the barycenter of the system's material points, that is, he came to the grounds of barycenter's coordinates, which turned out to be projection ones [20]. Thus, mechanic conception of barycenter acquired an abstract interpretation within projection geometry. Furthermore, GMP-strategy can have a lot of variations, for example:

1). With the help of barycenter's coordinates is given the interpretation of Hardy-Weinberg's law in the genetic population [21,22] and on this ground, a power interdisciplinary direction in the form of mathematical genetics has appeared [23];

2). In the end of the $X X^{\text {th }}$ century, a famous american specialist in the field of psychology of the arts R.Arnheim, propounded a thesis [24], according to which, the psychology of professional artists differs by rather intuitively sensitive color perception and as a result, they, anyhow, see the distribution of color shades on the pictorial field as a balanced one. This thesis is proved by the experience within the conception about the colorimetric barycenter of paintings, developed in the works [25-28], where it is introduced through mapping:

$$
\operatorname{Im} \times F \rightarrow W,
$$

which to every point of the pictorial image $I m$, depending on its color $F$, is brought to conformity with a non-negative number from $W$ set, which is designated the colorimetric mass of this point. The mapping (21) determines structural colorimetric of a pictorial work. The colorimetric barycenter of the picture, with the posi- tion of which the compositional peculiarities of a given picture are connected, can then be calculated on the basis of the well-known mechanics formulas. As investigations have shown [25-28], the colorimetrical barycenter is a balancing color point of a pictorial work and this fact has been proved by the formation of barycenter ensemble for the large collection of paintings. For this, barycenter ensemble coordinates of pictures are mapped on the unit square and dotting image of ensemble received like this gives an idea about barycenter dispersion relatively to central position. Figure 1 shows such colorimetric barycenter ensemble from 1174 pictures of painters of the $\mathrm{XX}^{\mathrm{th}}$ (white dot indicates the average position of the barycenter for the ensemble). It is evident, that artists representing a variety of compositional genres in many cases try to avoid significant deflections from equilibrium of colorimetric mass in the picture. This GMP-strategy within the conception of colorimetric barycenter represents an important component in teaching mathematics in the field of humanity education [29].

In pedagogics GMP-strategy is carried out on the basis of cybernetic conception [30]. Objectively, it is caused by the fact that in the field of didaktikos, pedagogics is based on the theory of cognitive processes, which realize transformation and transfer of information from generation to generation. Cybernetics promotes the development of pedagogical science helping solve arising contradictions between its content and form not only through experience, but also within the category of morphism with the help of modeling and optimization of pedagogical processes. The realization of cybernetic conception while making up the fundamental theory of mathematical models in order to regulate effectively cognitive processes in teaching comes from information nature of pedagogical processes. The control in this case can go on through aim influence on quantitative or qualitative aspects of information, realized in the teaching

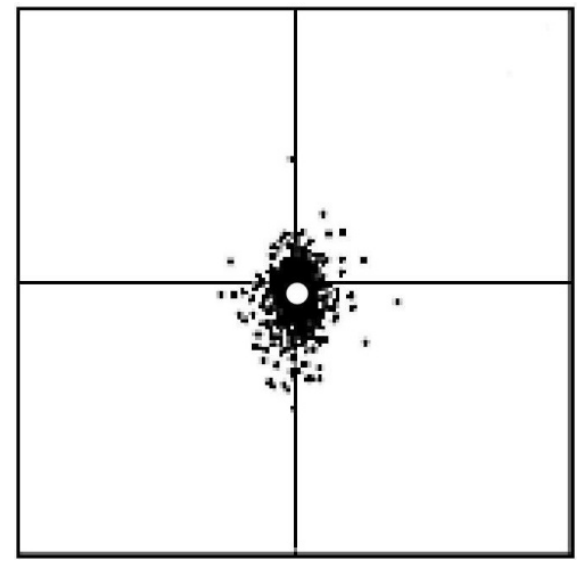


Figure 1. Colorimetric barycenter ensemble from 1174 pictures of painters of the XXth century.

process.

Models, describing the control by cognitive processes through aim influence on quantitative information aspect of corresponding educational content, are formed on the basis of metric functions, which, in this case, have an explicit mathematical form. The procedure of optimization in these models has a universe character, as abstract quantitative information measures are in its ground and the optimal control in this case leads to the improvement of the systems organization of the teaching process through the search of optimal configuration of information nets and flows in this process according to minimum criterion of information entropy. The class of basic models, the control of which is carried out according to the quantitative measures of information, includes: Socrat's dialogue, testing, class-lesson system of teaching, organization of group cooperation during the teaching process and the procedure of subject planning of the teaching process [31-34]. Given models make up the basis of a number of information technologies, which have been approved in the teaching process. In particular, at optimization of group cooperation in the teaching process the observations have shown the rise of progress standards of education contingent up to $20-25 \%$.

Models, describing the control of cognitive processes through aim influence on qualitative (semantic) information aspect of educational content, are formed within accepted model of knowledge interpretation. In accepted cognitological model (point 2) the system of knowledge is introduced within informal axiomatic theory in the form of semantic net. This net is properly metrized and is characterized by a definite system of coverings in point 3 . It makes possible to introduce net parameters of optimization, controlling qualitative aspects of the knowledge system under consideration (points 3-6). To the class of basic models, the control of which is carried out by influence on information semantic aspect, models of formation of education content, creative pedagogics and realization of teaching on interdicipline level are included. The criteria of such control optimization are formulated in points 3;4 and at optimization of deductive conclusion leads to reducing of volume of analyzed information within criteria (13),(14), and at optimization of creative processes they are controlled by criterion of significance (18). These theoretical statements are confirmed not only by experienced data of Tables 1, 2, but also are effectively realized by the author in his teaching work while training teachers of mathematics at Saratov State University after N.G. Chernyshevski (Russia) since 1997.

\section{CONCLUSIONS}

As a matter of fact, GMP-strategy based on Pareto-optimization (18) is treated as a control by definite random process $T h(S ; t)$, modeling the creative search while developing the space $T h(S)$. This search turns to be more effective, if it comes from more significant positions. This thesis is acknowledged not only by theoretical premises (points 3-6) and by the success in solving Hilbert's problems (Table 2), but by the whole process of historical mathematical development. It is difficult to overrate Pithagor's theorem in Euclid's geometry, Desargues's and Pascal's theorems in projective geometry, Euclid's algorithm in the theory numbers, Viete's theorem in the algebra of polynomial, Cayley's theorem in the theory of groups and so on.

Nowadays the significance of specified states is actual as well. Recently, in the work [35] within the framework of GMP-strategy for determining of Pythagorean triples, a special matrix transformation semigroup's transformation of primitive pairs has been made up. Thus, a close link between Pithagor's theorem and Euclid's algorithm is stated and original approaches to the solution of the oldest mathematical problem about the power of a set of twin primes numbers. It is important to say that, except the original mathematical results, an effective training to the methods of the mathematical creation takes place within the framework of GMP-strategy.

The possibilities of GMP-strategy are not limited only by the control of creative processes in the sphere of mathematical education. In the morphism's category, GMPstrategy is spread on the level of interdisciplinary teaching. Thus, the integration of mathematical knowledge in the field of natural and humanitarian sciences takes place. In this case, GMP-strategy realizes a certain variant of common scientific methodology (for example, canon or centrism). This variant is axiomatized and then theoretical model of the object or phenomenon is formed. It is important to say, that in the process of interdisciplinary GMP-strategy, effective training to the methods of mathematical creation takes place. So, the original reflexive conception in the fields of creative pedagogics is realized on the basis of GMP-strategy.

\section{ACKNOWLEDGMENTS}

The author thanks Alaitseva V. V., an English teacher of Gymnasia №5 (Saratov, Russia), for the help in translating this work paper.

\section{REFERENCES}

[1] Galilei, G. (1964) Il Saggiatore. Opere. Salani, Firenze.

[2] Newton, I. (1946) Mathematical Principles of Natural Philosophy. The Universitet of California Press, Berkley. 
[3] Boltzmann, L. (1909) Wissenschaftliche abhandlungen. Leipzig.

[4] Wiener, N. (1961) Cybernetics (or control and communication in the animal and machine). The MIT Press, New York.

[5] Shannon, C.E. (1948) A mathematical theory of communication. Bell System Technical Journal, 27(3), 379-423.

[6] Henry, P. and Lazarsfeld, N.W. (1966) Readings in mathematical social science. In: P. F. Lazarsfeld and N. W. Henry Eds., A Collection of Articles, Science Research Associates, Chicago.

[7] Rosenblatt, F. (1958) The perception: A probabilistic model for information storage and organization in the brain. Psychological Review, 65(6), 386-408.

[8] Feigenbaum, E.A. (1963) Computers and thought. In: E. A. Feigenbaum and J. Feldman Eds., A Collection of Articles, McGraw-Hill Book Co., New York, 477-523

[9] Anderson, J.R. (1983) The architecture of cognition. Harvard University Press, Cambridge, Massachusetts, USA.

[10] Hopfild, J.J. (1982) Neural networks and physical systems with emergent collective computational abilities. Proceedings of the National Academy of Sciences of USA, 79(8), 2554-2558.

[11] Brusilovsky, P. (1996) An intelligent tutoring system onWorld-Wide Web. Proceedings of the 3th International $W W W$ Conference, Fraunhofer Institute for Computer Grafics, Darmstagt, 42-45.

[12] Firstov, V.E. (2006) The semantic model and optimization trough mathematical knowledge formation and propagation. Vestnik Saratov State Technical University, 3(14), 34-43.

[13] Rashevsky, N. (1955) Live, information theory and topology. The Bulletin of Mathematical Biophysics, 17(3), $25-78$.

[14] Kolmogorov, A.N. [in Russian: Колмогоров А. Н. (1965) Три подхода к определению понятия "количество информации”. Проблмь передачи информации. Т.1, вып.1, сc.3-11].

[15] Firstov, V.E. (2006) The stochastic model by the information space of deductive theory formation and optimization of the research work in mathematics. Vestnik Saratov State Technical University, 4(17), 13-21.

[16] Harris, T.E. (1963) The theory of branching processes. Springer-Verlag, Berlin-Göttingen-Heidelberg.

[17] Glaser, R. (1984) Education and thinking: The role of knowledge. American Psychologist, 39(2), 93-104.

[18] Hilbert, D. (1900) Mathematische probleme. Nachr. Ges. Wiss. Göttingen, 253-297.

[19] Moulin, H. (1988) Axioms of cooperative decision making. Cambridge University Press, Cambridge-New YorkNew Rochelle-Melbourne-Sydney.
[20] Möbius, A.F. (1885) Der barycentrische calcul. Bd. 1, Gesammelte Werke, Leipzig.

[21] Hardy, G.H. (1908) Mendelian proportions in a mixed population. Science, 28(706), 49-50.

[22] Weinberg, W. (1908) Über den Nachwies der Vererbung beim Menschen. Jahreshefte des Vereins for Vater- landische Naturkunde in Würtemberg, 64, 368-382.

[23] Crow, J.F. and Kimura, M. (1970) An introduction in Population Genetics Theory. Harper and Row, New York.

[24] Arnheim, R. (1988) The power of the center. A study of composition in the visual arts. University of California Press, Berkeley.

[25] Firstov, V.V., Firstov, V.E. and Voloshinov, A.V. (2005) Conception of colorimetric barycenter in painting analysis. Proceedings of the International Congress on Aesthetics, Creativity and Psychology of the Arts, Moscow, 2005, 258-260.

[26] Firstov, V.V., Firstov, V.E. and Voloshinov, A.V. (2006) The concept of colorimetric barycenter in group analysis of painting. Culture and Communication: Proceedings of the XIX Congress International Association of Empirical Aesthetics, Avignon, France, 2006, 439-443.

[27] Firstov, V. V., Firstov, V. E., Voloshinov, A. V. and Locher, P. (2007) The Colorimetric Barycenter of Paintings. Empirical Studies of the Arts, 25(2), 209-217.

[28] Firstov, V.E., Firstov, V.V. and Voloshinov, A.V. (2008) The concept of colorimetric barycenter and visual perception of the color balance center in painting. Proceedings of the XX Biennial Congress of the International Association of Empirical Aesthetics, Chicago, USA, 2008, 273-277.

[29] Firstov, V.E. (2009) About teaching math in humanitarian specializations and occupations in IHE. Higher Education Today, 2, 82-84.

[30] Firstov, V.E. (2009) Cybernetic concept of current educational process. Higher Education Today, 3, 66-68.

[31] Firstov, V.E. (2007) Dialogue education: Cybernetic aspect. Vestnik Saratov State Technical University, 4(28), 135-145.

[32] Firstov, V.E. (2008) Informational conception of optimization of group cooperation in teaching. Vestnik Saratov State Technical University, 3(34), 105-109.

[33] Firstov, V.E. (2008) The concept of defeveloping training of L.S. Vygotsky, pedagogics of cooperation and cybernetics. Yaroslavl Pedagogical Bulletin, 3(56), 98- 104.

[34] Firstov, V.E. (2009) Expert systems and information conception in developing training. Yaroslavl Pedagogical Bulletin, 1(58), 69-73.

[35] Firstov, V.E. (2008) A special matrix trasformation semigroup of primitive pairs and the genealogy of pythagorean triples. Mathematical Notes, 84(2), 263-279. 